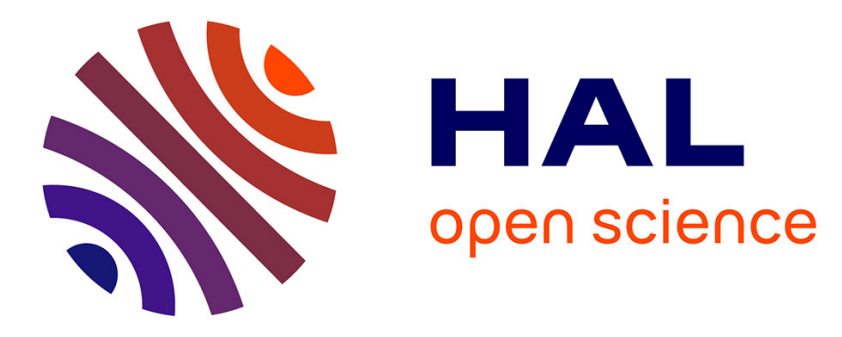

\title{
Amplification et émission paramétriques à l'échelle subpicoseconde dans un cristal organique: - application à la spectroscopie infra-rouge
}

J. Zyss, I. Ledoux, J. Badan, J.L. Oudar, J. Etchepare, D. Hulin, A. Migus, A. Antonnetti

\section{To cite this version:}

J. Zyss, I. Ledoux, J. Badan, J.L. Oudar, J. Etchepare, et al.. Amplification et émission paramétriques à l'échelle subpicoseconde dans un cristal organique: - application à la spectroscopie infra-rouge. Revue de Physique Appliquée, 1987, 22 (10), pp.1229-1238. 10.1051/rphysap:0198700220100122900 . jpa-00245673

\section{HAL Id: jpa-00245673 https://hal.science/jpa-00245673}

Submitted on 1 Jan 1987

HAL is a multi-disciplinary open access archive for the deposit and dissemination of scientific research documents, whether they are published or not. The documents may come from teaching and research institutions in France or abroad, or from public or private research centers.
L'archive ouverte pluridisciplinaire HAL, est destinée au dépôt et à la diffusion de documents scientifiques de niveau recherche, publiés ou non, émanant des établissements d'enseignement et de recherche français ou étrangers, des laboratoires publics ou privés. 


\title{
Amplification et émission paramétriques à l'échelle subpicoseconde dans un cristal organique : application à la spectroscopie infra-rouge
}

\author{
J. Zyss $(*)$, I. Ledoux $(*)$, J. Badan $\left({ }^{*}\right)$, J. L. Oudar $(*)$, J. Etchepare $\left({ }^{+}\right)$, D. Hulin $\left(^{+}\right)$, \\ A. Migus $\left(^{+}\right)$et A. Antonnetti $\left(^{+}\right)$ \\ (*) Centre National d'Etudes des Télécommunications (LA CNRS 250), 196, avenue Henri-Ravera, \\ 92220 Bagneux, France \\ $\left({ }^{+}\right)$Laboratoire d'Optique Appliquée, Ecole Polytechnique - ENSTA, 91120 Palaiseau, France
}

(Reçu le 4 décembre 1986, accepté le 4 mars 1987)

\begin{abstract}
Résumé. - Des impulsions optiques de forte puissance crête et de durée inférieure à la picoseconde sont obtenues par amplification paramétrique d'un continuum de lumière blanche dans un nouveau cristal organique, le NPP (pour N-(4-nitrophényl)-(L)-prolinol). Un lien est établi entre ce phénomène et les propriétés, tant microscopiques que macroscopiques, du NPP. La nécessité d'une révision du concept et de la terminologie traditionnels $d^{\prime}$ ' accord de phase non critique " est démontrée au vu des contraintes spatiotemporelles qu'impose l'observation d'effets non linéaires en régime impulsionnel ultra-rapide. C'est ainsi que sont dégagées les notions mieux adaptées au présent contexte d'« accord de phase $\lambda$-non critique » et ses implications discutées. Une nouvelle méthode spectroscopique autorisant une résolution temporelle inférieure à la picoseconde est issue du dispositif de mesure du NPP précédemment utilisé : la cellule d'eau émettant le continuum est simplement remplacée par l'échantillon dont la luminescence peut être étudiée dans l'intervalle 1,0-1,6 $\mu \mathrm{m}$. Cette technique nouvelle appelée PASS (pour l'anglais « Parametric Amplification and Sampling Spectroscopy »), est appliquée à l'étude d'une structure de type "Puits Quantique Multiple » à 1,44 $\mu \mathrm{m}$. L'utilisation future de matériaux organiques dans des dispositifs de traitement du signal infra-rouge est évoquée en conclusion.
\end{abstract}

\begin{abstract}
High peak power subpicosecond optical pulses have been generated by parametric amplification of a white light femtosecond continuum in a new organic crystal, N-(4-nitrophenyl)-L-prolinol (NPP). The relation between this observation and the structural properties, both at microscopic and macroscopic levels of NPP are exposed. Special emphasis is put on the renewal of the traditional concept of non-critical phasematching in view of the present requirements linked to the observation of ultrafast subpicosecond nonlinear phenomena. The notions of $\theta$-non critical and $\lambda$-non critical phase-matching together with their implications are exposed. A new method for time-resolved luminescence spectroscopy with subpicosecond resolution is simply derived as an extension of the NPP nonlinear measurement set-up where a previously used infra-red emitting continuum water cell is simply replaced by any sample of interest with luminescence in the 1.0-1.6 $\mu \mathrm{m}$ range. This new technique, defined as PASS, a tag for Parametric Amplification and Sampling Spectroscopy, is demonstrated at $1.44 \mu \mathrm{m}$ in a InGaAs-InAlAs multiple quantum well structure. The future of nonlinear organic materials in infra-red optical signal processing systems is briefly discussed in conclusion.
\end{abstract}

\section{Introduction.}

L'étude de la luminescence résolue en temps à l'échelle subpicoseconde dans le proche infra-rouge d'échantillons tels que les semiconducteurs ne peut être menée de façon tout à fait satisfaisante à l'aide de techniques connues. Le besoin de renouvellement de ces techniques est pressant pour des signaux de photoluminescence de faible énergie et de domaine spectral situé au-delà du très proche infra-rouge c'est-à-dire de 1 à $1,6 \mu \mathrm{m}$. En effet, la caméra à balayage de fente est spectralement limitée au 
visible et au très proche infra-rouge avec une résolution temporelle de l'ordre au mieux de quelques picosecondes. L'autre solution, que constituent les cellules Kerr optiques, permet un échantillonnage subpicoseconde de la luminescence grâce à un effet non linéaire cubique à faible rendement ce qui en limite l'application aux luminescences intenses. Signalons enfin l'addition de fréquences, l'un des faisceaux étant un laser ultra-bref, l'autre le signal de luminescence: le faisceau résultant, à la fréquence somme, a toutefois une énergie limitée par le rendement, également faible, du phénomène non linéaire quadratique mis en jeu. La méthode proposée ici se fonde sur l'amplification paramétrique et l'échantillonnage temporel du signal (PASS pour «Parametric Amplification and Sampling Spectroscopy ») et permet l'étude à l'échelle subpicoseconde de très faibles signaux de 0,9 à $1,6 \mu \mathrm{m}[1,2]$. Cette méthode utilise de façon essentielle les propriétés non linéaires d'un nouveau cristal organique, le NPP, [3] dans une configuration d'amplificateur paramétrique en simple passage. Après avoir présenté les principales caractéristiques structurelles du NPP et leur lien avec ses propriétés non linéaires, la configuration expérimentale de la spectroscopie PASS sera introduite comme un prolongement naturel de l'étude des propriétés non linéaires du NPP. La démonstration de l'efficacité de ce nouveau type de spectroscopie se fera sur la luminescence d'une structure à puits quantique multiple (MQWS) constitué d'alternance InGaAs-InAlAs.

\section{Relation entre la structure du NPP et son efficacité non linéaire quadratique.}

La variété des matériaux organiques, susceptibles de donner lieu par synthèse à toutes sortes de structures que l'on sait relier, par des modèles physiques maintenant éprouvés, à leurs propriétés optiques non linéaires a conduit au concept d'«ingénierie moléculaire » [4]. Des règles bien établies permettent d'optimiser, tant au niveau moléculaire que cristallin, la réponse non linéaire quadratique d'un cristal moléculaire, pour une plage spectrale d'utilisation donnée. On peut ainsi, en théorie, définir un cristal «optimal » et essayer, en pratique, de s'en rapprocher le plus possible. C'est ainsi que le NPP s'est avéré être, dans la famille des composés analogues à la paranitroaniline, proche de cet optimum structurel. Sa structure moléculaire (Fig. 1) comporte classiquement un système aromatique d'électrons $\pi$ fortement polarisables en interaction dissymétrique avec un groupement donneur (le prolinol) et accepteur (nitro) d'électrons. Ce type de structure, couramment appelée «diode moléculaire », possède une non-linéarité élevée, de l'ordre de quelques $10^{-29}$ u.e.s., supérieure de deux ordres de grandeur à celle de composés saturés aliphatiques

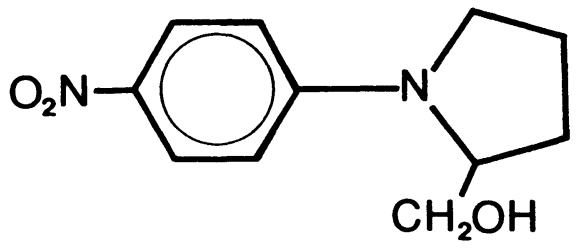

Fig. 1. - Structure moléculaire du NPP, le groupement prolinol agissant comme électrodonneur et le groupement nitro comme électroaccepteur sur le système aromatique conjugué.

[Molecular structure of NPP : the prolinol group acts as an electron donor while the nitro group acts as an electroacceptor on the aromatic electronic system.]

de mêmes dimensions. La polarité élevée de ce type de composés, dits à transfert de charge, favorise toutefois en général l'établissement d'une structure centrosymétrique, par le jeu des interactions dipôledipôle. Une structure centrosymétrique annule en effet les coefficients des tenseurs de rang impair associés à une propriété physique, ce qui est le cas des non-linéarités quadratiques. Dans le cas du NPP, deux facteurs vont toutefois s'opposer à cette tendance : le premier est la présence d'un carbone chiral [5] dans le groupement prolinol, dérivé de la proline, acide aminé naturellement lévogyre. Un cristal alors constitué d'unités moléculaires de même isomérie optique ne saurait en effet être géométriquement invariant par symétrie plane ou ponctuelle. Le second facteur est la présence d'un groupement alcool susceptible d'établir des liaisons hydrogène entre molécules du réseau cristallin qui articulent les molécules les unes par rapport aux autres sans que puisse se faire sentir la tendance à la centrosymétrie induite par des interactions dipôle-dipôle de plus faible énergie. De fait, la structure cristallographique du NPP est monoclinique $\left(P 2_{1}\right)$ et l'angle entre les deux molécules de la même maille cristalline et l'axe binaire est de $58,6^{\circ}$, s'approchant de la valeur optimale de $54,7^{\circ}$ qui optimise le coefficient $d_{21}$. Ce dernier est seul à permettre, au contraire du coefficient $d_{22}$, un accord de phase par biréfringence. Cet angle optimal résulte trivialement de l'optimisation, dans le cadre d'un modèle de gaz orienté, du coefficient angulaire reliant le coefficient $d_{21}$ à la non-linéarité $\beta$ supposée unidimensionnelle le long de l'axe des deux atomes d'azote soit :

$$
d_{21}=N f\left(\cos ^{2} \theta\right)(\sin \theta) \beta
$$

où $N$ est le nombre de molécules par unité de volume et $f$ un facteur de champ local qu'il n'est pas utile d'expliciter ici. L'encadré inférieur de la figure 3 montre, à la demi-translation de maille près le long de $Y$, une vue simplifiée de cette structure cristalline. La structure cristalline est caractérisée par le parallélisme entre les plans moléculaires 


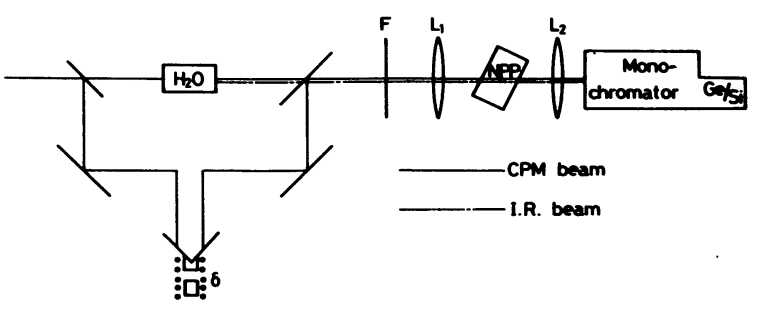

Fig. 2. - Dispositif de mesure des propriétés non linéaires du NPP. Le laser CPM à $620 \mathrm{~nm}$ génère un continuum dans la cellule d'eau et déclenche un gain paramétrique dans l'échantillon de NPP placé sur un support orientable. Une ligne à retard permet de réaliser, dans le cristal, la corrélation de l'émission infra-rouge de l'eau avec le signal du laser de pompe. Le dispositif de détection comporte un monochromateur et une photodiode de Germanium ou de Silicium. Un tel dispositif, suivant le dispositif de filtrage $F$ choisi, permet d'étudier la génération de second-harmonique, l'émission et l'amplification paramétrique dans le NPP.

[Measurement set-up of the nonlinear properties of NPP. The CPM laser at $620 \mathrm{~nm}$ generates a white light continuum in the water cell and triggers a parametric gain in the NPP sample set on a goniometric mount. A delay line allows for the cross-correlation in the crystal of the $I-R$ emission with the pump laser. The detection set-up consists of a monochromator and a Germanium or Silicium slow-response time photodiode whichever is relevent to the wavelength range of interest. According to the nature of the filter(s) F, such a set-up allows as well for the determination of SHG, parametric emission or amplification characteristics.]

moyens coïncidant à peu près avec le plan des cycles aromatiques et les plans cristallins $\langle 101\rangle$. Des liaisons hydrogène intramoléculaires s'établissent entre d'une part l'atome d'hydrogène du groupement alcool d'une molécule donnée et les deux oxygènes $\mathrm{du}$ groupement nitro de la molécule voisine du même plan cristallin. Il en résulte une structure lamellaire, avantageuse du point de vue optique comme on le verra dans la partie 3 mais pouvant induire, en l'absence de précautions mécaniques lors de la croissance ou de la manipulation (découpe ou polissage) une certaine tendance au clivage, parallèlement aux mêmes plans $\langle 101\rangle$.

Comme on le discutera en appendice, deux types d'accord de phase sont souhaitables selon qu'on désire une acceptance angulaire ou une acceptance spectrale maximales : dans le premier cas, classiquement appelé non critique et que l'on préférera appeler ici $\theta$-non critique [4], la pente de la courbe $\lambda(\varphi), \varphi$ étant l'angle d'incidence sur le cristal des faisceaux en interaction, $\lambda$ la longueur d'onde du rayonnement fondamental. Le second cas, appelé $\lambda$ non critique où $\lambda(\varphi)$ a une pente verticale, est de grand intérêt dans le cas d'impulsions ultra-brèves, donc à grande largeur spectrale $\Delta \lambda$. Une variation angulaire faible $\Delta \varphi$ de la configuration relative

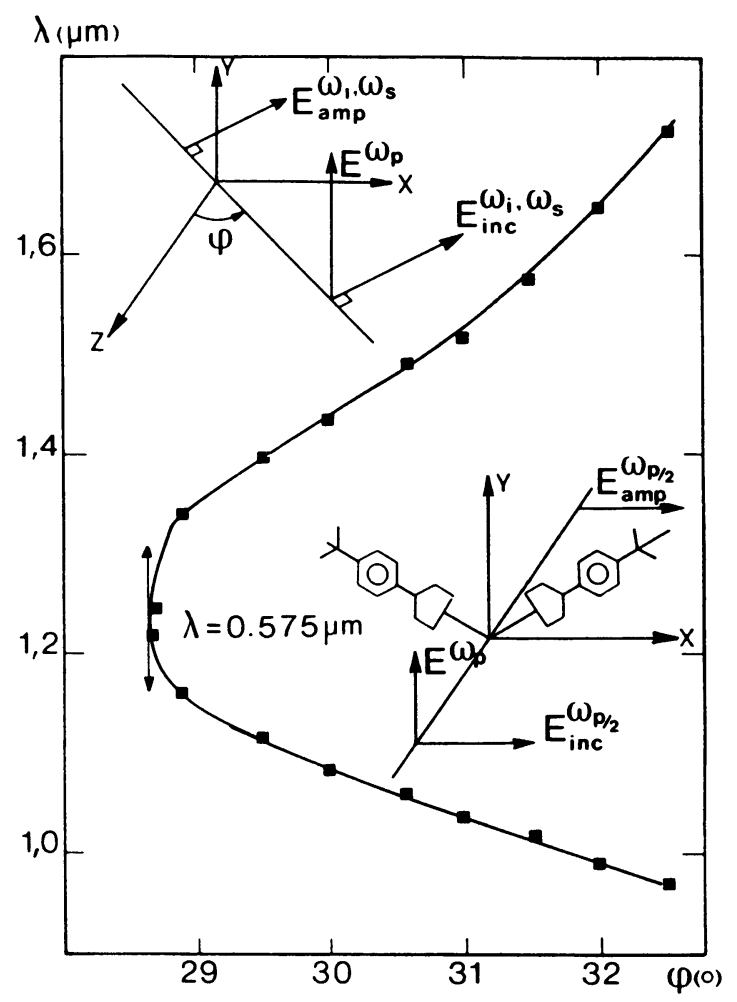

Fig. 3. - Courbe reliant les longueurs d'onde de signal et de complémentaire à l'angle externe d'incidence du faisceau de pompe correspondant à l'accord de phase colinéaire pour l'amplification paramétrique dans le NPP. L'encart supérieur représente une configuration quelconque des plans de polarisation du signal ou du complémentaire, incident sur, ou émis par le cristal. Un cas particulièrement intéressant est décrit dans l'encart inférieur : il correspond à l'accord de phase $\theta$-non critique à $\lambda_{\mathrm{p}}=$ $575 \mathrm{~nm}$.

[This curve relates the signal and idler wavelength to the external incidence angle of the pump laser on the NPP crystal corresponding to a collinear phase-matching configuration for parametric amplification. The upper insert displays a general configuration of the incoming and/or out-going signal and idler waves. A case of special interest is pointed-out in the lower insert corresponding to $\theta$-non critical phase matching configuration at $\lambda_{p}=575 \mathrm{~nm}$.]

cristal-faisceaux incidents permet de parcourir une plage d'accordabilité $\Delta \lambda$ considérable.

Cette deuxième situation se présente naturellement dans le cas des cristaux moléculaires de type paranitroaniline ou voisins comme on le verra dans l'appendice. La non-linéarité, mesurée en solution par génération de second-harmonique sous champ dans l'acétone, du NPP [6] soit $(44 \pm 9) 10^{-30}$ u.e.s., en projection le long de l'axe dipolaire de la molécule, lui-même sensiblement confondu avec l'axe reliant les deux atomes d'azote aboutit, après sommation cristalline selon un modèle de gaz orienté (cf. Eq. (1)), au même ordre de grandeur que la détermination expérimentale. Celle-ci a été effectuée sur une lame mince monocristalline de NPP de 
$11 \mu \mathrm{m}$ [7], longueur sensiblement inférieure à la longueur de cohérence pour ce type de matériaux, par optimisation angulaire du coefficient $d$ efficace de génération de second-harmonique et aboutit à une valeur de $d_{21}$ de l'ordre de $190 \times 10^{-9}$ u.e.s. à $1,06 \mu \mathrm{m}$, valeur la plus forte rapportée à ce jour, à notre connaissance, pour un coefficient anisotrope.

\section{Caractérisation du NPP à l'échelle subpicose- conde.}

La source utilisée est un laser à colorant dit à "verrouillage de mode et collision d'impulsions" (CPM) produisant des impulsions de $100 \mathrm{fs}$ à $620 \mathrm{~nm}$, amplifié à un taux de répétition de $10 \mathrm{~Hz}$ par passage dans quatre cellules à colorant pompées par un laser YAG : $\mathrm{Nd}^{3}+$ doublé en fréquence [8]. L'énergie des impulsions de sortie est de l'ordre de $1 \mathrm{~mJ}$ et le faisceau séparé en deux. Un des faisceaux (la pompe) est polarisé le long de l'axe $Y$ du cristal qui èst aussi axe de rotation du montage. L'autre faisceau est focalisé dans une cellule d'eau de façon à générer, par auto-modulation de la phase, un continuum de lumière blanche d'une durée de $180 \mathrm{fs}$ dont le spectre s'étend du visible au-delà de $1,6 \mu \mathrm{m}$. Les longueurs d'onde situées en dessous de $1 \mu \mathrm{m}$ sont éliminées à l'aide d'un filtre Schott RG 1000. Le faisceau de pompe et le continuum infra-rouge se recouvrent dans le cristal, séparés par un intervalle de temps variable grâce à une ligne à retard montée sur un moteur pas à pas (voir Fig. 3). Le faisceau de pompe est réduit à un diamètre transverse de $1 \mathrm{~mm}$, l'énergie atténuée à $1 \mu \mathrm{J}$, ce qui correspond à une intensité incidente de $1 \mathrm{GW} \mathrm{cm} \mathrm{cm}^{-2}$. Le signal I.R. est focalisé dans le cristal à un diamètre inférieur à celui du faisceau de pompe. L'expérience consiste à amplifier dans le cristal la portion spectrale du continuum $\lambda \pm \Delta \lambda$ issue de l'eau correspondant par la relation d'accord de phase $\lambda(\varphi)=0$ à l'orientation relative $\varphi$ cristal-faisceaux incident. On initialise l'expérience en engendrant le second-harmonique de $\lambda_{\mathrm{d}}=1,24 \mu \mathrm{m}$ ce qui restitue la longueur d'onde de pompe $\lambda_{\mathrm{p}}=620 \mathrm{~nm}$. On est alors dans la configuration dite de dégénérescence pour l'amplification paramétrique où $\lambda_{\mathrm{c}}=\lambda_{\mathrm{s}}=\lambda_{\mathrm{d}}=2 \lambda_{\mathrm{p}}$ (longueurs d'onde du complémentaire et du signal confondues). On ajuste alors le retard temporel $\tau$ de façon à ce que le signal et la pompe arrivent simultanément dans le cristal, c'est-à-dire en maximisant le signal amplifié vu au travers d'un système de détection (monochromateur et photodétecteur ajustés à la longueur d'onde $\lambda_{\mathrm{d}}$ ). On dispose alors du point à tangente verticale de la courbe d'amplification paramétrique d'allure grossièrement parabolique reliant $\varphi$ à $\lambda_{\mathrm{s}}$ et $\lambda_{\mathrm{c}}$. On poursuit l'expérience en décalant la longueur d'onde affichée sur le monochromateur et en cherchant l'angle d'incidence sur le cristal optimisant l'amplification du signal. L'itération de cette procédure conduit à l'angle d'accord de phase et à la longueur d'onde correspondante. La courbe d'accord de phase se situe dans le plan $Z X$, le cristal agissant, pour chaque angle $\varphi$, comme un filtre amplificateur pour les fréquences correspondantes $\lambda_{\mathrm{s}}$ et $\lambda_{\mathrm{c}} \mathrm{du}$ signal et de son complémentaire. La pente quasi verticale de la courbe d'accord de phase (représentée en Fig. 3) dans le domaine spectral considéré augmente d'autant l'acceptance spectrale de l'amplificateur (de 800 à $1000 \AA$ autour de la dégénérescence) tout en minimisant le désaccord des vitesses de groupe (cf. appendice). La figure 4 représente la

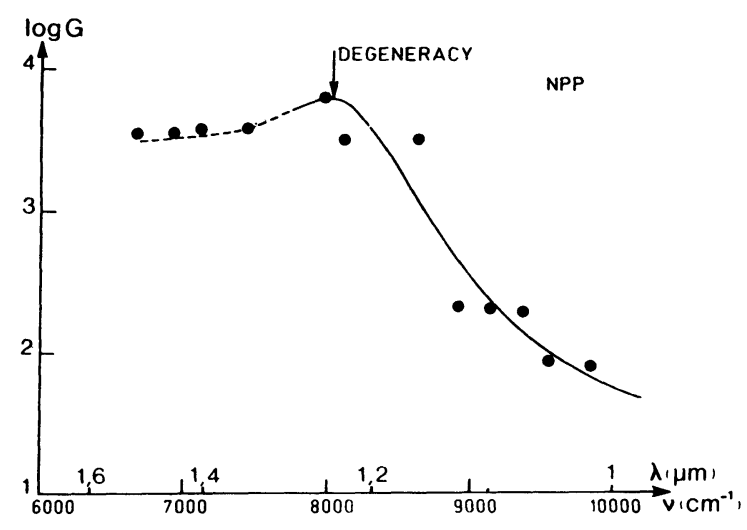

Fig. 4. - Dispersion en longueur d'onde du gain paramétrique montrant un maximum à la dégénérescence $\left(\lambda_{\mathrm{d}}=1,24 \mu \mathrm{m}\right)$. L'aspect asymétrique de la courbe est due à la nature du système de filtrage spectral (voir texte).

[Wavelength dispersion of the parametric gain showing a maximum at degeneracy $\left(\lambda_{\mathrm{d}}=1.24 \mu \mathrm{m}\right)$. The non-symmetric shape of the curve results from the specifity of the filtering system adapted here (see text).]

dépendance en fréquence du gain. Le gain est maximal comme il se doit aux environs de la dégénérescence $(\lambda=1,24 \mu \mathrm{m})$ et de l'ordre de $G=10^{4}$. Tout le continuum situé au-dessus de $1 \mu \mathrm{m}$ est incident sur le cristal : il en résulte que le monochromateur étant réglé à la même fréquence $\lambda_{s}$, la diode mesure non seulement le signal amplifié à la même fréquence $\lambda_{\mathrm{s}}$ mais aussi le complémentaire du signal engendré par l'amplification de la longueur d'onde $\lambda_{c}$ incidente qui se retrouve être à la fréquence $\lambda_{s}$. C'est le cumul des deux signaux que l'on observe sur la figure 4 et qui en explique l'aspect dissymétrique : en effet l'énergie du continuum audelà de $1,45 \mu \mathrm{m}$ étant faible son apport, dans la partie haute fréquence de la courbe de gain (en dessous de 1,24 $\mu \mathrm{m}$ ), est plus faible que pour la partie basse fréquence de la courbe. Ceci explique la surévaluation du gain au-delà de $1,4 \mu \mathrm{m}$ : ce phénomène peut toutefois se révéler avantageux si l'on souhaite émettre des impulsions d'intensité crête 
élevée dans ce domaine spectral. L'expression du gain en régime non saturé [9] permet d'estimer, à la longueur d'onde de dégénérescence, la valeur du coefficient $d$ efficace (soit $d_{\text {eff }}$ ):

$$
d_{\mathrm{eff}}=\frac{\log (4 G)}{E_{\mathrm{p}}}\left(\frac{n_{\mathrm{s}} n_{\mathrm{c}}}{\omega_{\mathrm{s}} \omega_{\mathrm{c}}}\right)^{1 / 2} \frac{\varepsilon_{0} c}{l}
$$

où $l$ est la longueur d'interaction (ici de l'ordre de $1,5 \mu \mathrm{m}), E_{\mathrm{p}}$ l'amplitude du champ de pompe incident et $n_{\mathrm{s}}$ (resp. $n_{\mathrm{c}}$ ) se rapporte à l'indice de réfraction du signal (resp. du complémentaire). On trouve une valeur de $d_{21}$ de l'ordre de $100 \times 10^{-9}$ u.e.s. $\left(3,5 \times 10^{-22}\right.$ S.I. $)$ à comparer à la valeur mentionnée à la partie 2 de $190 \times 10^{-9}$ u.e.s., cette dernière mesure ayant été effectuée sur des couches minces parfaitement monocristallines. Le désaccord peut s'expliquer par les désorientations cristallines au long de l'échantillon de $1,5 \mathrm{~mm}$ d'épaisseur. La solution de divers problèmes liés à la croissance des échantillons par la méthode de Bridgman-Stockbärger [10], tels que la relaxation des contraintes lors de l'ouverture de l'ampoule, favorisant l'apparition de clivages ou de dislocation et la présence d'un certain taux d'impuretés résiduelles, devraient permettre d'améliorer ces performances et de rapprocher la longueur effective utilisée dans l'expression [2] de la longueur physique de l'échantillon. Par ailleurs, les pertes par diffusion dans le cristal ne sont pas tout à fait négligeables et une certaine saturation du gain limite la validité de l'expression (1). Une autre limitation du gain pourrait provenir du désaccord des vitesses de groupe aux longueurs d'onde de pompe $\lambda_{\mathrm{p}}$ et de signal $\lambda_{\mathrm{d}}$ à la dégénérescence $\left(\lambda_{\mathrm{d}}=2 \lambda_{\mathrm{p}}\right)$.

Comme on le montrera en appendice, les vitesses de groupe des deux ondes, projetées sur la direction commune de leurs vecteurs d'onde sont rigoureusement égales ce qui assure le recouvrement temporel des impulsions interagissantes, au prix d'un certain désaccord du recouvrement spatial transverse (l'angle de walk-off), au demeurant négligeable sur la faible longueur d'interaction considérée ici, soit $1,5 \mathrm{~mm}$. La confirmation expérimentale de cette propriété s'obtient en retardant le signal de pompe par rapport au signal incident du continuum et en enregistrant l'énergie du signal amplifié en fonction du retard variable (voir encart de la Fig. 6) : il s'agit d'un type original de corrélation croisée obtenue par amplification paramétrique et qui diffère du schéma classique d'autocorrélation par génération de second-harmonique [11]. A faible intensité de signal incident, hors du régime de saturation du gain, l'élargissement temporel du signal amplifié par rapport au signal incident apparaît inférieur à $100 \mathrm{fs}$. Lorsqu'on accroît l'intensité du signal incident, l'élargissement temporel provenant de la saturation du gain et de l'amplification correspondante du pied

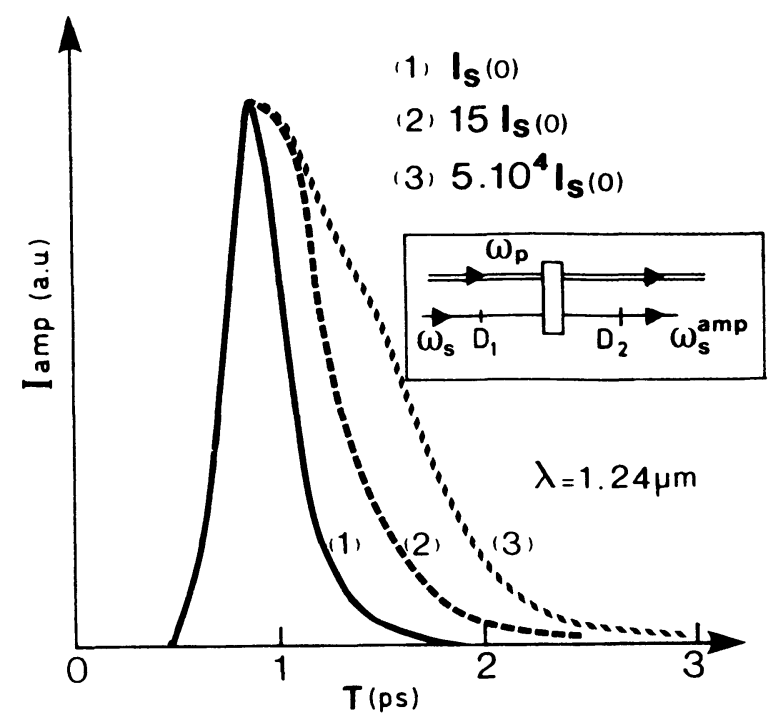

Fig. 5. - Intensité des signaux amplifiés mesurée en fonction du retard entre la pompe et le signal incident pour des valeurs différentes de l'intensité du signal incident $I_{\mathrm{S}}(0)$. L'élargissement temporel de l'impulsion témoigne de la saturation du gain aux fortes intensités de signal incident.

[Intensity of the amplified signal as a function of the delay between pump and signal at various values of the incident intensity $I_{\mathrm{S}}(0)$. The time-broadening of the pulse confirms the saturation of the gain at high intensities.]

à faible intensité de la courbe (1) de la figure 5 peut aller jusqu'à une valeur de 1 ps. Une expérience de génération de second-harmonique, décrite par ailleurs, a permis de mettre en évidence un accord de phase $\theta$-non critique à $\lambda=1,15 \mu \mathrm{m}$ correspondant, en l'absence de «walk-off », à la maximisation de l'acceptance angulaire du dispositif, lorsque pompe et signal se propagent le long de l'axe diélectrique $Z$ (voir encart inférieur de la Fig. 3). La longueur d'onde de pompe de l'amplification paramétrique correspondante est de $0,575 \mu \mathrm{m}$, à l'intérieur du domaine d'émission d'un colorant laser classique, la Rhodamine 6G. Dans cette configuration $d_{\text {eff }}=$ $d_{21}$, en l'absence de tout facteur de projection angulaire. Les polarisations représentées dans l'encart inférieur de la figure 3 sont alors toutes trois, pompe, signal et complémentaire, dans le plan $\langle 101\rangle$, mettant alors pleinement en jeu le rôle des électrons du système conjugué. Le plan de polarisation de ces trois ondes coïncide en effet avec le plan des électrons polarisables responsables de l'effet non linéaire.

L'émission paramétrique a pu être observée pour une intensité de pompe supérieure à $3 \mathrm{GW} \mathrm{cm}^{-2}$, la largeur de bande émise s'étendant de 1 à $1,4 \mu \mathrm{m}$. Une caractérisation spectrale et temporelle plus fine des signaux émis ou amplifiés, utilisant deux cristaux de NPP pompés consécutivement, fera prochainement l'objet d'une publication. A ce stade, le NPP 


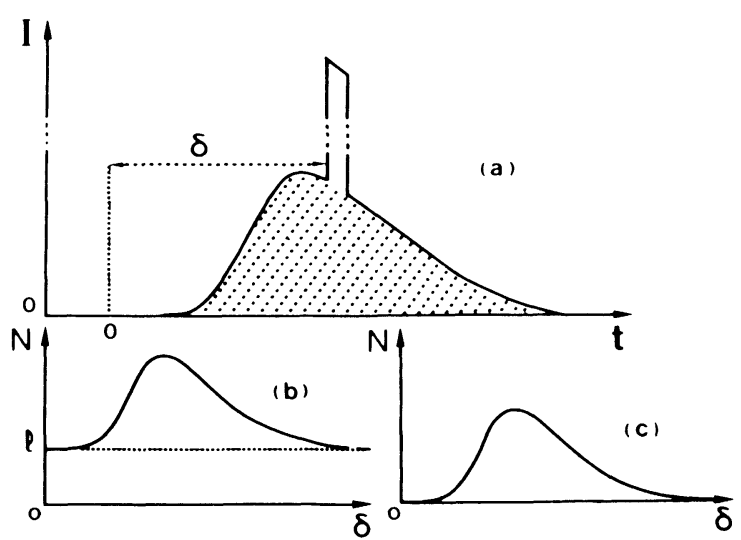

Fig. 6. - Schéma de principe de la spectroscopie «PASS ». La courbe (a) représente la luminescence après son passage dans le cristal de NPP. Seule la fraction de la luminescence coïncidant temporellement avec le faisceau de pompe est amplifiée d'un facteur $10^{4}$. Le retard $\delta$ entre la pompe et le signal peut être ajusté de façon à explorer toute la durée de la luminescence. La courbe (b) représente le signal intégré recueilli sur la photodiode (nombre $N$ de photons) en fonction du retard $\delta$ à une longueur d'onde de luminescence intéressante. La ligne de base $l$ correspond à la valeur de l'aire pointillée en (a). Le contraste $C$, défini par l'exp. (4), traduit l'écart moyen entre la courbe (b) et la ligne de base. En (c) figure la réponse de la photodiode à la longueur d'onde complémentaire.

[Principle of the «PASS " spectroscopy. Curve (a) stands for the luminescence after interaction with the pump in the NPP crystal. Only that fraction of the luminescence with coincides in time with the pump beam is amplified by a factor of $10^{4}$. The delay $\delta$ between pump and signal can be adjusted so as to explore the whole duration of the luminescence. Curve (b) represents the signal (total number $N$ of photons) such as integrated by the photodiode at the luminescence wavelength of interest, as a function of the delay $\delta$. The base-line value $l$ is the value of the dotted area in curve (a). The contrast ratio $C$, such as defined by exp. (4), is a quantitative assessment of the possibility to make-out between curve (b) and the baseline. Curve (c) represents the photodiode response at the idler wavelength.]

apparaît être un bon candidat pour la réalisation de sources infra-rouges femtosecondes accordables. Des puissances allant du MW dans la configuration de l'émission paramétrique par simple passage jusqu'au GW après amplification dans un second cristal de plus grande dimension transverse sont en effet possibles.

\section{Echantillonnage temporel et amplification para- métrique infra-rouge dans un cristal de NPP (spec- troscopie PASS).}

Le principe même des expériences de caractérisation des effets non linéaires dans le NPP à l'échelle subpicoseconde s'avère adapté à la résolution à la même échelle de temps de la luminescence d'échantillons quelconques, ceci jusqu'à 1,6 $\mu \mathrm{m}$. Supposons en effet que l'on substitue, au moins en principe, à la cellule d'eau génératrice du continuum, un échantillon luminescent dans l'infra-rouge dans des conditions similaires de pompage. Le mélange dans le cristal de NPP avec un retard variable du signal de luminescence et du signal de pompe va alors permettre l'échantillonnage temporel et l'amplification du signal de luminescence. Comme on l'a vu en 3 , un gain de $10^{4}$ peut être obtenu au travers de $1,5 \mathrm{~mm}$ de NPP dans des conditions d'orientation et de polarisation correspondant à l'accord de phase aux alentours de la dégénérescence $(\lambda=1,24 \mu \mathrm{m})$. Le système de détection comporte une photodiode à temps de réponse long qui mesure une quantité proportionnelle au nombre total de photons collectés. Si l'on appelle $(\tau)$ la durée moyenne de la luminescence et $n$ le nombre moyen de photons incidents par seconde sur la photodiode, le signal enregistré en l'absence de pompe est en fait un bruit $B$ et vaut :

$$
B=n\langle\tau\rangle \text {. }
$$

La pompe, incidente sur le cristal entre les instants $\delta$ et $\delta+\tau_{\mathrm{p}}$ ( $\delta$ retard pompe-signal, $\tau_{\mathrm{p}}$ durée du signal de pompe), va amplifier avec un gain $G$ le signal de luminescence et s'ajouter à la contribution précédente $B$ pour fournir le signal $S$ donné par:

$$
S=n \tau_{\mathrm{p}} G+n\langle\tau\rangle \text {. }
$$

On fait ici l'approximation que le même nombre $n$ de photons incidents en moyenne par seconde sur le cristal vaut pour l'intervalle $\left(\delta, \delta+\tau_{\mathrm{p}}\right)$ et pour la durée $\langle\tau\rangle$ de la luminescence : ce serait en toute rigueur le cas pour un signal de luminescence rectangulaire de durée $\langle\tau\rangle$. Le rapport signal sur bruit de l'expérience $S / B$ vaut alors :

$$
\frac{S}{B}=1+G \frac{\tau_{\mathrm{p}}}{\langle\tau\rangle}=1+10^{4} \frac{\tau_{\mathrm{p}}}{\langle\tau\rangle}
$$

Pour une durée de pompe de 100 fs et une durée de luminescence de l'ordre de $100 \mathrm{ps,} \mathrm{typique} \mathrm{pour}$ les puits quantiques, le rapport $S / B$ vaut 10 , ce qui permet d'extraire, dans des conditions satisfaisantes, la seule partie amplifiée de la luminescence du bruit de fond que constitue la luminescence intégrée. Le rapport signal à bruit est d'autant meilleur que le temps de luminescence est bref par rapport au temps de pompe tout en maintenant un gain élevé, conditions qui se trouvent réalisées dans l'expérience décrite ci-après. La figure 6 illustre le principe de la spectroscopie PASS. Dans la mesure où l'on évite de saturer le gain, on suit sans déformation, avec une résolution temporelle qui n'est limitée que par la durée de l'impulsion de pompe, la montée puis la décroissance de la luminescence. Les intensités de 
luminescence sont en général assez peu intenses pour que soit évité ce phénomène de saturation. Dans toutes les applications, nous avons pu vérifier la linéarité du signal de sortie en fonction du signal d'entrée. Notons aussi que le bruit de fond, représenté par la droite horizontale en pointillé de la figure $6 \mathrm{~b}$, peut être supprimé en observant le signal complémentaire de la luminescence amplifiée : en effet à tout photon de luminescence amplifié correspond un photon à la longueur d'onde complémentaire vérifiant la relation de conservation de l'énergie $\omega_{\mathrm{p}}=\omega_{\mathrm{s}}+\omega_{\mathrm{c}}$. La configuration expérimentale convertit le retard $\delta$ en différence de chemins optiques. Le premier faisceau, dit d'excitation, est focalisé dans l'échantillon après avoir parcouru un trajet optique $l_{1}$. La luminescence résultante est collectée au moyen d'une lentille de grande ouverture et refocalisée après un trajet $l_{1}^{\prime}$ dans le cristal de NPP. Le second faisceau (faisceau de pompe) parcourt un trajet optique $l_{2}$ variable avant de recouvrir spatialement le signal de luminescence dans le cristal non linéaire. L'aire du faisceau est de l'ordre de $1 \mathrm{~mm}^{2}$ et son énergie atténuée jusqu'à environ $10 \mu \mathrm{J}$ ou moins, ce qui correspond à une intensité incidente de quelques $\mathrm{GW} \mathrm{cm} \mathrm{cm}^{-2}$. La référence de temps initial étant l'instant où l'impulsion excitatrice atteint l'échantillon, la portion amplifiée du spectre de luminescence est émise avec un retard $\delta$ donné par :

$$
\delta=\left[l_{2}-\left(l_{1}+l_{1}^{\prime}\right)\right] / c
$$

$c$ étant la vitesse de la lumière. La résolution temporelle du phénomène d'amplification paramétrique (cf. Fig. 5) est inférieure à 200 fs en l'absence de saturation du gain. Un microordinateur contrôle la ligne à retard (trajet $l_{2}$ ). L'instant d'incidence du signal de pompe sur l'échantillon est déterminé par l'amplification d'un signal infra-rouge réfléchi sur l'échantillon, de façon à «simuler » une luminescence instantanée.

Ce dispositif expérimental se prête à la caractérisation du temps de réponse des dispositifs à semiconducteur dans le proche infra-rouge, parmi lesquels les structures à puits quantiques multiples (MQWS) présentent un grand intérêt, en particulier pour le calcul optique. L'étude préliminaire présentée ici porte sur des alternances de puits et de barrières de InGaAs-InAlAs [12] dont la transparence, de l'ordre de $1,5 \mu \mathrm{m}$, correspond aux longueurs d'onde des systèmes de télécommunication par fibre optique. Les structures à puits quantique GaAs-GaAlAs, dont le domaine de transparence est de l'ordre de $0,8 \mu \mathrm{m}$ sont mieux connues. La figure 8 montre la résolution temporelle de la luminescence à $1,44 \mu \mathrm{m}$ d'une structure à puits quantique InGaAs-InAlAs à $15 \mathrm{~K}$, excitée par une impulsion d'une durée de $100 \mathrm{fs}$ à $620 \mathrm{~nm}$ incidente sur l'échantillon à l'instant $t=0$. Le signal de photoluminescence se manifeste

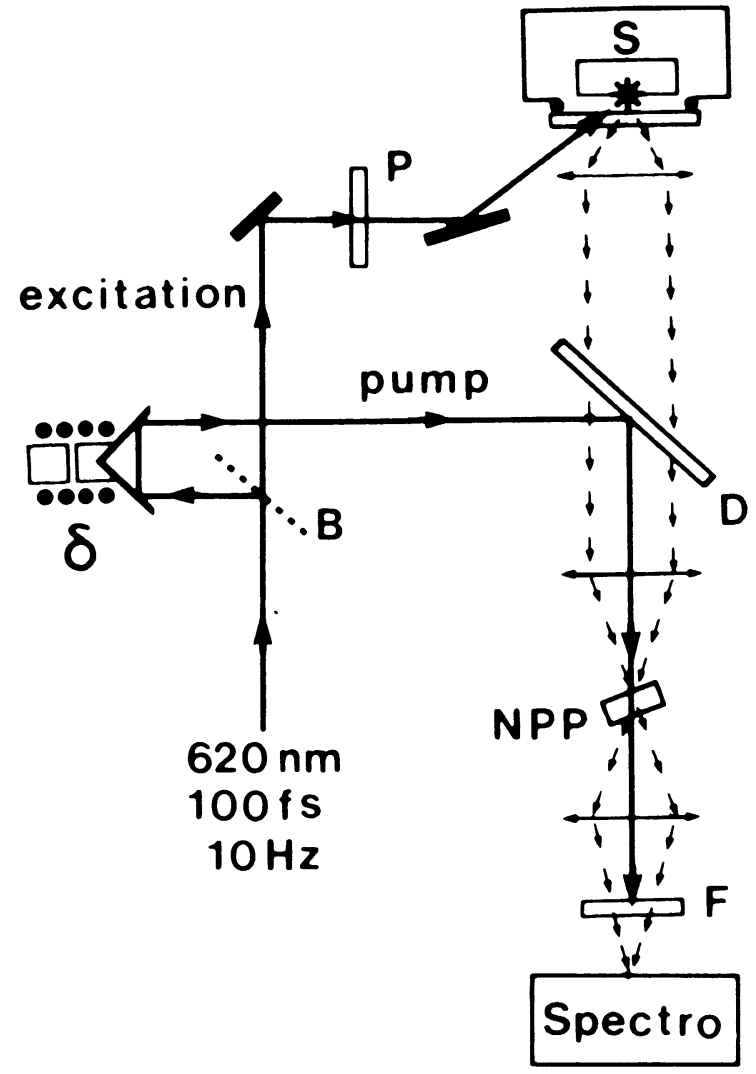

Fig. 7. - Configuration expérimentale de la spectroscopie « PASS ». B est une lame séparatrice, $P$ une lame demionde permettant d'ajuster la polarisation, $S$ l'échantillon (situé ici dans un cryostat à Hélium), D une lame dichrö̈que réfléchissant le faisceau de pompe et transmettant le faisceau infra-rouge, et $F$ un filtre rejetant le faisceau de pompe.

[Experimental wavelength on PASS spectroscopy : B is a beamsplitter, $P$ a half-wave plate to adjust the polarization, $S$ the sample of interest (possibly as here in a Dewar), D a dichroic plate reflecting in the visible and transmitting in the infra-red range of relevance here, and $F$ a filter meant to reject the pump beam.]

avec un retard d'environ 15 ps par rapport à l'excitation. Contribuent à ce retard le transport des paires électron-trou photoexcitées à $2 \mathrm{eV}$ au travers de la couche tampon InAlAs surmontant les puits quantiques, suivi de leur recombinaison radiative dans les puits quantiques. L'interprétation théorique plus approfondie de ces observations est en cours.

\section{Conclusion.}

Au-delà du NPP, d'autres monocristaux, plus colorés mais également transparents dans le rouge (longueur d'onde de pompe) peuvent être conçus à partir de molécules à plus forte réponse non linéaire [4]. Ces molécules qui comportent, par exemple, deux cycles aromatiques au lieu d'un cycle unique (cas des dérivés du stilbène) ou, plus généralement, dont le 


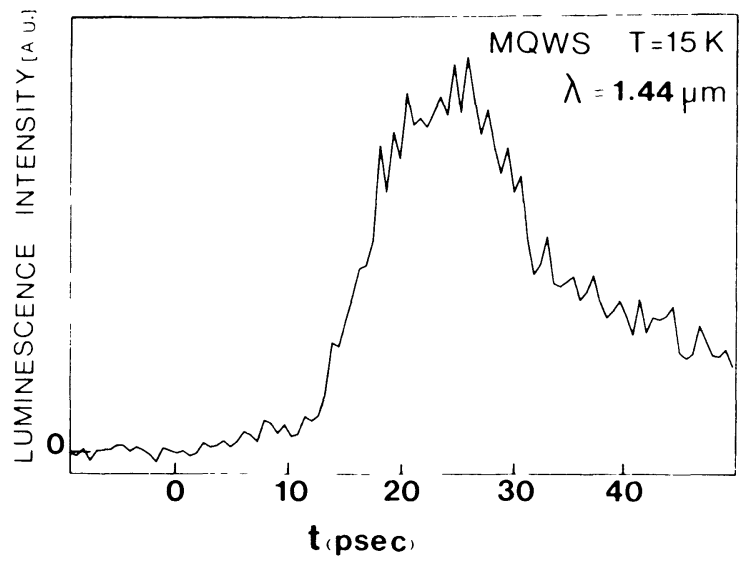

Fig. 8. - Luminescence résolue en temps à $1,44 \mu \mathrm{m}$ d'une structure à puits quantique multiple InGaAs-InA1As à $15 \mathrm{~K}$ excitée avec un faisceau de pompe à $0,62 \mu \mathrm{m}$. L'instant de référence $t=0$ est obtenu par amplification de l'émission du continuum à $1,44 \mu \mathrm{m}$ réfléchie à la surface de l'échantillon de façon à simuler une réponse instantanée de l'échantillon à l'excitation.

[Time resolved luminescence at $1.44 \mu \mathrm{m}$ of a Multiple Quantum Well (MQW) structure InGaAs-InAlAs excited by a pump beam at $0.62 \mu \mathrm{m}$. The reference time $t=0$ is obtained by amplifying the emission of the continuum at $1.44 \mu \mathrm{m}$ as simply scattered on the sample surface so as to simulate instantaneous luminescence response.]

système d'électrons polarisables est de plus grande extension (polyphényls ou polyènes disubstitués à nombre élevé d'unités monomériques), sont au moins dix fois plus non linéaires [13] au niveau microscopique (tenseur $\beta$ ). A structure cristalline identique à celle du NPP, il est raisonnable d'anticiper, pour ces futurs monocristaux, des gains en régime non saturé dépassant de deux ordres de grandeur ceux mesurés dans cette étude. A ces progrès de type structurels s'ajouteront progressivement ceux de l'optique guidée dans ce type de matériaux : le confinement dans des guides plans ou des fibres constitués de matériaux organiques hautement non linéaires est susceptible de venir encore exalter considérablement les gains effectifs et leur intégration dans un dispositif de traitement du signal optique n'est pas, à terme, un objectif irréaliste [14].

A ce stade nous avons démontré l'utilisation nécessaire de matériaux à forte-non-linéarité pour l'échantillonnage et l'amplification de signaux infrarouges. Le terme «box-car» optique a pu être proposé pour définir l'usage du NPP dans l'expérience de spectroscopie PASS décrite ici. S'il ne s'agit pas à proprement parler de calcul optique, c'est bien un procédé original de traitement du signal optique que permet l'efficacité de matériaux nouveaux. Leurs possibilités dans ce contexte, apparaissent prometteuses et encore largement inexplorées.

\section{Annexe.}

Nous nous référerons dans ce qui suit à la génération de second-harmonique de type I $\left(e^{\omega}+e^{\omega} \rightarrow o^{2 \omega}\right)$ situation correspondant, tant du point de vue angulaire que spectral, au cas de l'amplification paramétrique à la dégénérescence dans le NPP (pompe à la fréquence $2 \omega$, signal et complémentaire à la même fréquence $\omega$ ). Dans l'approximation de l'onde plane l'intensité du second-harmonique est donnée par une fonction du type sinc $(\Delta k l / 2)$, où $\Delta k$ est la différence entre vecteurs d'ondes harmonique-ordinaire et fondamental-extraordinaire. La condition d'ouverture angulaire et spectrale peut alors s'exprimer :

$$
-1<\Delta k(\theta, \lambda) l<1 .
$$

Développée au premier ordre cette expression aboutit à :

$$
\begin{aligned}
& -l^{-1}<\left.\Delta \theta \frac{\partial(\Delta k)}{\partial \theta}\right|_{\theta_{0}, \lambda_{0}}+ \\
& +\left.\Delta \lambda \frac{\partial(\Delta k)}{\partial \lambda}\right|_{\theta_{0}, \lambda_{0}}<l^{-1}
\end{aligned}
$$

où $\Delta \theta=\theta-\theta_{0}$ et $\Delta \lambda=\lambda-\lambda_{0}$ correspondent respectivement aux désaccords angulaires et spectraux mesurés à partir des paramètres $\lambda_{0}$ et $\theta_{0}$ à la dégénérescence. L'acceptance spectrale (resp. angulaire) maximale est obtenue pour

$$
\left.\frac{\partial}{\partial \lambda}(\Delta k)\right|_{\theta_{0}, \lambda_{0}}=0 \quad\left(\text { resp. }\left.\frac{\partial(\Delta k)}{\partial \theta}\right|_{\theta_{0}, \lambda_{0}}=0\right) .
$$

La courbe d'accord de phase en génération de second harmonique étant donnée par

$$
\Delta k(\theta, \lambda)=0
$$

la condition $\frac{\partial}{\partial \theta}(\Delta k)_{\theta_{0}, \lambda_{0}}=0$ exprime que la tangente en $\theta_{0}$ à cette courbe dans le repère $\theta, \lambda$ est horizontale (cf. Fig. A.1). La permutation de $\theta$ et $\lambda$ aboutit à des considérations symétriques pour l'acceptance spectrale, la relation $\frac{\partial}{\partial \lambda}(\Delta \theta)_{\theta_{0}, \lambda_{0}}=0$ exprimant que la tangente à cette courbe est verticale. Il est alors légitime de parler de ces configurations en termes d'accord de phase angulairement non critique (ou $\theta$-non critique) et spectralement non critique (ou $\lambda$-non critique). Cette dernière condition aboutit à une relation entre les indices de réfraction donnée par :

$$
\left.2 \frac{\partial n^{\mathrm{e}}}{\partial \lambda}\right|_{\lambda}=\left.\frac{\partial n^{0}}{\partial \lambda}\right|_{\lambda / 2} .
$$

Appelant $\mathbf{u}$ et $\mathbf{u}_{1}\left(\operatorname{avec} \mathbf{u}_{2}=-\mathbf{u}_{1}\right)$ le repère polaire classique associé à l'angle $\theta$, on peut montrer que la vitesse de groupe d'une onde plane de fréquence $\omega$ 

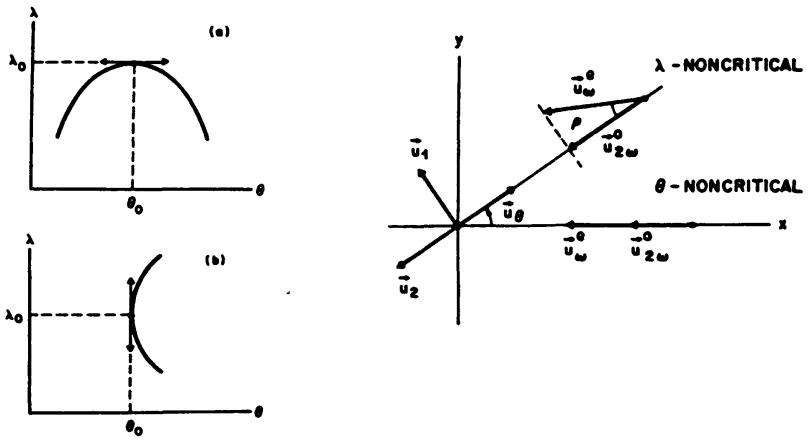

Fig. A.1. - (a) Courbes d'accord de phase à pente horizontale correspondant à l'accord de phase angulairement $\theta$-non critique. (b) Courbe d'accord de phase à pente verticale correspondant à l'accord de phase spectralement $\lambda$-non critique. Une configuration d'accord de phase $\lambda$-non critique implique la conservation de la vitesse de groupe le long de la direction commune des vecteurs d'onde. Dans la configuration $\theta$-non critique les vitesses de groupe sont parallèles à un axe principal diélectrique mais leurs modules diffèrent.

[(a) Phase-matching curve with horizontal slope corresponding to angular $\theta$-non critical phase matching. (b) Phase-matching curve with a vertical slope corresponding to spectral $\lambda$-non critical phase-matching. A $\lambda$-non critical phase-matching configuration entails the conservation of the group velocities of the interacting waves projected along the common wavevector orientation. In the $\theta$-non critical configuration, group velocities are parallel to a principal dielectric axis but their moduli may significantly differ.]

correspondant à l'indice de réfraction $\boldsymbol{n}$ et à l'angle de walk-off $\rho$ vaut :

$$
\mathbf{u}_{\omega}=\frac{c}{n+\omega\left(\frac{\partial n}{\partial \omega}\right)}\left(\tan \rho \mathbf{u}_{1}-\mathbf{u}_{2}\right) .
$$

La condition d'accord des vitesses de phases impliquant :

$$
n_{\omega}^{\mathrm{e}}=n_{2 \omega}^{0}
$$

les relations (A.3) et (A.5) permettent d'égaliser la composante sur $\mathbf{u}_{2}$ (ou $\mathbf{u}$ ) de $\mathbf{u}_{\omega}^{\mathrm{e}}$ et $\mathbf{u}_{2}^{0}{ }_{\omega}$ soit :

$$
\left(\mathbf{u}_{2 \omega}^{0}-\mathbf{u}_{\omega}^{\mathrm{e}}\right) \cdot \mathbf{u}=0 \text {. }
$$

Cette expression signifie qu'à l'angle de walk-off $\rho$ près, les vitesses de groupe de l'onde fondamentale et de l'onde harmonique sont égales dans la configuration $\lambda$-non critique. Il est à noter que la condition (A.3) a naturellement tendance à se vérifier pour les cristaux moléculaires du type NPP comme le montre la figure A.2. Une tendance analogue peut être relevée dans les cas du MAP [15] (méthyl-(2,4dinitrophényl)-amino-propanoate) et du POM [16] (3-méthyl-4-nitropyridine-1-oxide) où les courbes d'accord de phase déterminées expérimentalement ont, à partir de $1,4 \mu \mathrm{m}$ et au-delà, l'aspect vertical

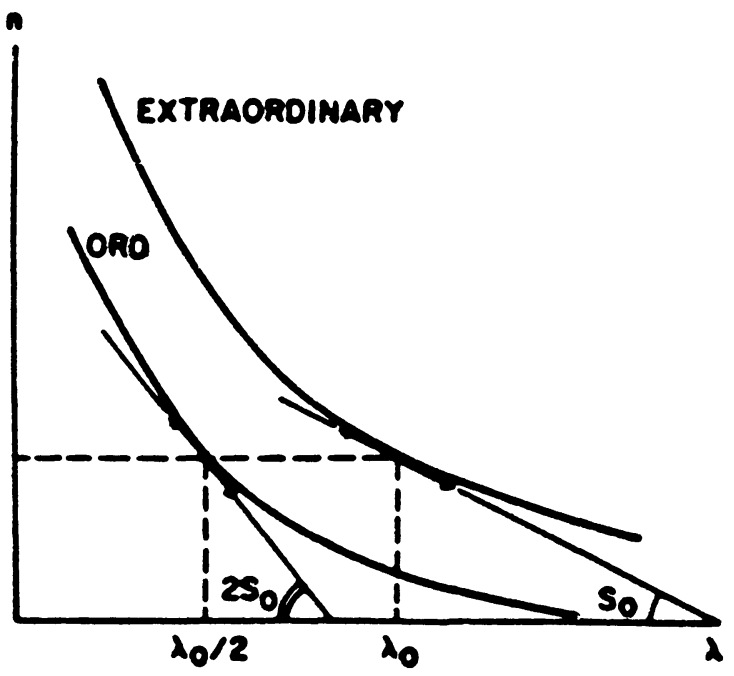

Fig. A.2. - L'existence d'un accord de phase non critique dans un cristal uniaxe à $\lambda=\lambda_{0}$ apparaît dans une configuration d'accord de phase de type I $\left(e^{\omega}+e^{\omega} \rightarrow 0^{2 \omega}\right)$ lorsque la valeur de la pente de la courbe de dispersion de l'indice ordinaire à la longueur d'onde harmonique vaut le double de celle correspondant à l'indice fondamental-extraordinaire. Une telle condition se trouve naturellement remplie dans les cristaux organiques tels que le NPP pour le domaine de longueur d'onde infra-rouge où les résonances visibles et infra-rouges compensent mutuellement leurs effets.

[In a uniaxial crystal, a non-critical type I phase-matching configuration $\left(e^{\omega}+e^{\omega} \rightarrow 0^{2 \omega}\right)$ occurs whenever the slope of the ordinary harmonic index dispersion curve comes close or equals twice that corresponding to the extraordinary fundamental. Such a condition is naturally met in organic crystals such as NPP in the infra-red domain where visible resonance contributions balance that of U.V. charge transfer related ones.]

caractéristique de l'accord de phase $\lambda$-non critique. Elle exprime en effet que dans le repère $[\lambda, n]$ la pente de la courbe représentant la dispersion de l'indice ordinaire aux faibles longueurs d'onde $\left(\lambda_{0} / 2\right)$ est nettement supérieure à la pente de la courbe représentant la dispersion de l'indice extraordinaire aux plus fortes longueurs d'onde $\left(\lambda_{0}\right)$, le rapport optimal étant 2 , comme l'exprime la relation (A.3). Cette tendance se justifie physiquement par la proximité à $\lambda_{2} / 2$ des résonances U.V., en particulier de l'état excité dit de transfert de charge (aux alentours de $450 \mathrm{~nm}$ pour le NPP), qui accentue fortement l'inclinaison de la courbe $n^{0}(\lambda)$. Dans un langage plus mathématique, l'influence du ou des pôles représentant les états U.V. dans une expression de type Sellmeier des indices introduit une exaltation résonante de l'indice de réfraction à $\lambda_{0} / 2$, si cette longueur d'onde se situe dans le visible. Par contre, à la longueur d'onde infra-rouge $\lambda_{0}$, l'effet des résonances U.V. ne se fait guère plus sentir et la dispersion des indices de réfraction tend à s'amoindrir. 


\section{Bibliographie}

[1] Ledoux, I., Zyss, J., Migus, A., Etchepare, J., Grillon, G. et Antonnetti, A., Appl. Phys. Lett. 48 (1986) 1564.

[2] Hulin, D., Migus, A., Antonnetti, A., Ledoux, I., Badan, J., Oudar, J. L. et Zyss, J., Appl. Phys. Lett. 49 (1986) 761.

[3] Zyss, J., Nicoud, J. F. et Coquillay, M., J. Chem. Phys. 81 (1984) 5160.

[4] Zyss, J. et Chemla, D. S., Quadratic Nonlinear Optics and Optimization of Second Order Nonlinear Response of Organic Molecules and crystals, in Nonlinear Optical Properties of Organic Molecules and Crystals, édité par D. S. Chemla et J. Zyss, 2 vols. (Academic Press, New York), 1987.

[5] ZYss, J. et Tsoucaris, G., Mol. Cryst. Liquid Cryst. 137 (1986) 303.

[6] Barzoukas, M., Zyss, J. et Morley, J., J. Opt. Soc. Amer. (JOSAB) 4, n 6 (1987) 977.

[7] Ledoux, I., Josse, D., Vidakovic, P. et ZYss, J., Opt. Eng. 25 (1986) 202.

[8] Migus, A., Antonetti, A., Etchepare, J., Hulin,
D. et Orszag, A., J. Opt. Soc. Am. B 2 (1986) 584.

[9] YARIV, A., Optical Electronics (Holt, Rinehard et Winston, New York), 1976.

[10] Badan, J., Hierle, R., Perigaud, A. et VidaKovIC, P., Growth and characterization of Molecular Crystals, in [4].

[11] IPPEN, E. P. et SHANK, C. V., Dans Ultrashort Light Pulses (Springer, Berlin, 1977) et WEINER, A. M., IEEE J. Quantum Electron. QE-19 (1983) 1276.

[12] Goldstein, L., Jean-Louis, A. M., Marzin, J. Y., Allovon, M., Allibert, C. et Gaillard, S., dans Proceedings of the International Symposium on GaAs and Related Compounds, Biarritz (1984) édité par B. de Cremoux (Adam Hilger, Bristol-Boston) 1985, p. 133.

[13] Oudar, J. L., J. Chem. Phys. 67 (1977) 446.

[14] ZYss, J., J. Mol. Electronics 1 (1985) 25.

[15] Oudar, J. L. et Hierle, R., J. Appl. Phys. 48 (1977) 2699.

[16] Zyss, J., Chemla, D. S. et Nicoud, J. F., J. Chem. Phys. 74 (1981) 4800. 